\title{
Kernos
}

Revue internationale et pluridisciplinaire de religion grecque antique

29 | 2016

Varia

\section{Dictionnaire du paganisme grec. Notions et débats autour de l'époque classique}

\section{Pierre Brulé}

\section{(2) OpenEdition \\ Journals}

Édition électronique

URL : http://journals.openedition.org/kernos/2436

DOI : 10.4000/kernos.2436

ISSN : 2034-7871

Éditeur

Centre international d'étude de la religion grecque antique

Édition imprimée

Date de publication : 1 octobre 2016

Pagination : 447-451

ISSN : 0776-3824

Référence électronique

Pierre Brulé, «Dictionnaire du paganisme grec. Notions et débats autour de l'époque classique », Kernos [En ligne], 29 | 2016, mis en ligne le 01 octobre 2016, consulté le 10 décembre 2020. URL : http://journals.openedition.org/kernos/2436 ; DOI : https://doi.org/10.4000/kernos.2436

Ce document a été généré automatiquement le 10 décembre 2020.

Kernos 


\title{
Dictionnaire du paganisme grec. Notions et débats autour de l'époque classique
}

\author{
Pierre Brulé
}

\section{RÉFÉRENCE}

Reynal SOREL, Dictionnaire du paganisme grec. Notions et débats autour de l'époque classique, Paris, Les Belles Lettres, 2015. 1 vol. $15 \times 21,5 \mathrm{~cm}, 513$ p. (Vérité des Mythes). ISBN :

978-2-251-38569-3

1 Les conditions et aussi la matière de cette recension sont particulières. Pour une fois, les sentiments ont eu bien de la peine à être muselés comme il convient selon les usages et les rigueurs qui président à cet exercice. La forme et le contenu s'en trouvent tout transformés jusqu'à manquer au devoir d'analyse du contenu notionnel d'un tel dictionnaire et jusqu'à laisser s'exprimer (un peu) l'ire du recenseur. À celui-ci d'apprécier l'intérêt, les qualités et défauts d'une œuvre, la couronner ou bien distribuer cartons jaunes ou rouges s'il les pense justifiés. Bien. Mais il peut arriver, rarement, qu'il ait subi auparavant un choc (comment le taire ici, et dans une telle revue ?) - c'est ce que j'ai ressenti à la lecture de ce titre, choc dont je ne suis pas encore remis. Paganisme! Quel mépris, ai-je d'emblée pensé ! À quelle sublimité doiton considérer se trouver pour traiter de la sorte une autre culture? Il faut sans doute n'avoir jamais imaginé jouer le rôle, banal puisque nous le partageons tous, d'être le païen d'un autre. Tout cela m'a longtemps bridé pour écrire cette appréciation: ce trouble me faisait craindre de mal contrôler mon computer. Même si j'espérais, ouvrant le livre, y trouver quelque raison pour faire mon autocritique.

2 J.-Fr. Mattéi, le préfacier de l'ouvrage, annonce d'emblée quel type d'éclairage y est utilisé pour éclairer des «notions » et des «débats» dans la religion grecque. Qu'ai-je écrit là ? La religion grecque? L'A. n'a pas souhaité l'introduire dans son titre ${ }^{1}$, ni dans 
son vocabulaire. Peut-être parce que, selon Mattéi, il s'agit moins d'y traiter d'une " religion » que de la vision " grecque de la condition humaine en matière de croyance religieuse $^{2}$, d'institution politique et de vie sociale» (p. 11). Curieuse phrase. Les Grecs de l'Antiquité n'aurait donc pas droit à une religion, mais à une « vision de la condition humaine ». J'avoue d'ailleurs que cette expression: "vision grecque de la condition humaine... » ne m'est pas absolument claire. Faut-il entendre "condition humaine » dans le sens que mes contemporains lui donnent ? Existerait-il " une vision grecque » de celle-ci ? Et où cette vision de l'humaine condition en matière religieuse serait-elle exprimée, et par qui?

3 Sous une forme qui ressemble à une sorte de confession, il annonce le programme et la méthode adoptés par l'A.: « Nous ${ }^{3}$ croyons parfois être débarrassés du point de vue chrétien, mais notre regard sur la Grèce demeure celui d'une culture qui s'est établie contre 4 un monde révolu qualifié de "païen" ( «nous» essayons de penser cette culture autrement que comme des chrétiens, mais nous n'y parvenons pas. Si je comprends bien, cela signifie que le fait de mettre la pensée religieuse grecque au gabarit monothéiste (le chrétien, bien sûr) la trahit, voire la dénature, la "dissout ». On ne peut que souscrire à un tel constat, bien des ouvrages sur la question, et celui-ci plus que d'autres, le prouvent. Mais en quoi l'exégète serait-il obligé de choisir un référent monothéiste ? Soit à comprendre une pensée religieuse polythéiste, qui pourrait nier que le meilleur comparatisme devrait être établi avec d'autres polythéismes?

4 Faute de pouvoir (savoir?) se dépouiller du regard chrétien, continue-t-il sans regret décelable, «nous » nous résolvons à penser non pas la religion mais le "paganisme » des Grecs en assumant un regard de chrétien, mieux, un regard de chrétien opposé à des gens et une culture qualifiés de "païens $»^{5}$. On tombe tristement des nues! Nous qui pensions être sortis de cette bataille vieille de deux millénaires! À peine au pied de l'opus, nous voici en pleine polémique culturelle!

5 Même s'ils anticipent sur son point de vue, et sans doute en accord avec lui, ce ne sont là que les mots d'un préfacier, laissons-le donc tranquille pour donner maintenant la parole à l'A. dans sa proclamation préalable (on ne peut pas dire qu'on ne l'attendait pas) où, selon ses propres mots, il veut régler incessamment la question de ce qu'il appelle un anachronisme, celle de l'emploi du mot " paganisme " ${ }^{6}$ (p. 15).

6 Pour lui tordre le cou, je commence par lui. Je me vois obligé de rappeler que le mot pagien ( $\mathrm{x}^{\mathrm{e}} \mathrm{s}$.), puis païen (vers 1100 ), est emprunté au latin paganus, " paysan», et son dérivé, "paganisme ", du latin d'église : paganismus. Il apparaît et se répand en Occident dans le contexte historique particulier du christianisme naissant. Plus nombreux dans les villes, les adeptes de cette religion, qui se considèrent comme éclairés, ajoutent une seconde macule au mépris habituels des urbains pour les glèbeux, en effet, ces paysans ont maintenu durant un temps variable des rites et des croyances anciennes dérivées des divers polythéismes antiques, devenus ridicules et hostiles à leurs yeux. Voilà pour l'origine probable du mot (l'A. ne cite pas les travaux de Chr. Mohrmann), mais ce qui compte plus, c'est son usage : un païen, c'est d'abord un polythéiste (on le dit souvent idolâtre ${ }^{7}$ ), puis, par un glissement qui n'est pas inattendu dû à la compétition entre les religions, le païen devient un hérétique, et les chrétiens appliquent le terme au judaïsme et à l'islam. Parallèlement à l'usage de "gentil » ou de "roumi ", rien de plus autocentré que la conception du monde à laquelle renvoie ce mot. Tous, chrétiens, juifs et musulmans, persuadés d'être dans le pieux, le juste, le 
vrai, font des autres des païens. Le lecteur se demande alors quel rapport entretient le paganisme avec ce dont parle le plus souvent ce livre : la religion grecque.

7 Il peut douter qu'il soit possible de travestir d'habits neufs, même le temps d'un livre, un mot dont le sens est si clair, si discriminant, il se dit que l'A. aura beau faire, le païen restera celui qui croit autrement et à autre chose, et le paganisme, la religion (inférieure) des autres ${ }^{8}$. Pourtant, privilège du penseur, j'admets bien sûr que sa fin justifie ses moyens, il peut se donner toutes les méthodes, les procédures d'élucidation qu'il juge efficaces - ce que le nôtre fait, d'ailleurs. La seule condition mise à cette liberté, c'est que la base sur laquelle chacune s'appuie soit suffisamment solide et clairement établie. Apprécions son nouveau paganisme.

"J'appelle "paganisme" " l'intrication de deux administrations : celle du sacré et celle de la cité ${ }^{10}$. Des citoyens d'un côté, des dieux de l'autre, et l'entretien de ceux-ci par ceux-là. C'est pourquoi cet ouvrage se concentre sur l'époque dite classique, sur ces deux siècles qui ne croyaient plus vraiment en des mortels demi-dieux sortis de l'âge 'homérique' et qui ignoraient encore les hommes divinisés conçus plus tard au sein de ce que nous appelons l'époque " hellénistique " (p. 15).

9 C'est important de poser les bons concepts. Mon malheur, c'est que dans cette définition, je tique à chaque terme ou presque. "Intrication»? Peut-être qu'imbrication a paru trop faible? Qu'on m'excuse, ai-je jamais rencontré ce mot? Pas chez Littré, ni dans le Grand Robert de 1970, mais (ouf !) je le trouve sur internet : « ce qui est emmêlé, enchevêtré »; j'y apprends qu'il est très utilisé à propos des phénomènes spécifiques de la mécanique quantique. Bien. Alors qu'est-ce qui est "emmêlé » dans la cité ? Deux «administrations ». Je re-tique. Mot imprécis, d'une certaine façon, "aventureux», que n'osent pas employer les spécialistes des institutions grecques parce qu'il convient mal à leur fonctionnement, c'est un à peu près. Ce qui ajoute encore à mes difficultés d'interprétation c'est la logique qui ferait dépendre la troisième phrase: "C'est pourquoi cet ouvrage se concentre sur l'époque dite classique... », des deux précédentes : "J'appelle "paganisme " l'intrication... » et « Des citoyens d'un côté, des dieux de l'autre... »

10 Quoi qu'il en soit, le "paganisme » de l'A. trouve son fondement, peut-être son essence, dans cette intrication de deux administrations, celle des citoyens et celle de la cité, auquel s'ajoute "l'entretien de ceux-ci [les dieux] par ceux-là [les citoyens].» «Entretien », encore un mot difficile. Qu'entendre par l' «entretien» des dieux? Que les Grecs honorassent, craignissent leurs dieux, mais que leur capital souci fût de les entretenir m'avait échappé. À moins qu'il ne s'agisse de l'emploi le plus commun du terme, c'est-à-dire dans son sens matériel; cet entretien pourrait, par exemple, faire allusion aux frais des cultes, comme ce que coûteraient ailleurs en d'autres temps de construire une cathédrale ou de fabriquer des vêtements sacerdotaux puis d'entretenir tout ça. Ce n'est certes pas ainsi que lesdits citoyens grecs antiques définissaient la nature de leurs rapports aux dieux, même si, par ailleurs, l'entretien (le maintien dans leur intégrité) des hiera de toutes sortes leur causait bien des soucis. Pour trouver la raison de cet «entretien » chez l'A. peut-être faut-il supposer que ce mot l'autoriserait à user du terme « administration " pour évoquer cette « intrication »... Bref, je n'ai pas très bien compris ce que désigne le "paganisme " grec à propos duquel l'A. entend épingler « notions » et « débats ».

11 Ces prolégomènes qui définissent un néo-paganisme difficile à relier au paganisme tel qu'on l'entend partout, se termine par la question de la chronologie: pour quelle 
époque ce dictionnaire est-il valable ${ }^{11}$ ? L'A. laisse choir ceux des Grecs qui croyaient aux demi-dieux sortis de l'âge " homérique » selon sa formule. Probablement pense-t-il à des Achille, ou à des Thésée, des Héraclès. Mais à qui fera-t-on croire que la pensée de ces demi-dieux (malheureuse expression!), leur présence dans les mythes et les rites, expriment l'essence de la religion des Grecs d'avant les guerres médiques? Et, inversement, que le changement de période les aient fait disparaître? Qu'un Solon, qu'un Pisistrate confondissent Ajax et Arès, Hélène et Aphrodite? Pourquoi utiliser cette façon négative et contournée pour dire qu'il ne parlera pas de la religion grecque de l'époque archaïque? Serait-ce parce que là, les philosophes dont il fait grande pâture, sont moins diserts? Sa manière de définir sa chronologie ignore le fait que les mêmes dieux et de nombreuses pratiques rituelles de l'époque classique existent à l'époque archaïque, qu'elles persistent à l'époque hellénistique et encore, au Haut Empire, certaines jusqu'à l'Antiquité tardive. Si elle en a ajouté, les hommes divinisés inclus, l'époque hellénistique n'a modifié le panthéon qu'en doses infinitésimales, comme l'époque classique l'avait fait au panthéon archaïque. Il en va de même des rapports des communautés aux theia pragmata («La cité n'est pas morte à Chéronée ! ", nom d'une pipe). Produits on ne peut plus humains, les religions sont des êtres historiques, elles évoluent, les monothéismes aussi.

L'A. poursuit l'exposé de son programme: "Le paganisme grec 'classique' est un ensemble de pratiques sans théoricien. » On ne le chicanera pas sur « un ensemble de pratiques». En effet, si la religion grecque n'est pas que cela, si elle est aussi représentation du monde et des rapports entre nature et surnature, humain et divin, elle l'est néanmoins dans une large mesure, et il est exact que dans son cadre on y voit en effet des hommes s'interroger sur leurs dieux et sur leurs pratiques. Toutefois, on concèdera qu'à la différence des monothéismes occidentaux, les Grecs polythéistes ne nous ont pas légué de ces longs et précis et acharnés commentaires rédigés pendant des millénaires sur leurs propres rites et leurs croyances. Il conviendrait de s'interroger sur cette relative absence. L'A. omet d'établir un rapprochement entre ce qu'il dit de l'absence de théoricien, qu'il dénonce, et celle du dogme qu'il souligne aussi (p. 17) ${ }^{12}$. Une bonne dose de logique plaiderait pour que le premier prétendu déficit se nourrisse du second.

Je ne quitte pas encore cette page 15 pour deux dernières remarques. Selon l'A., sur une balance des valeurs, il faudrait, d'un côté, plaindre ces Grecs de vivre une "piété inquiète... démunie de toute certitude » (pauvres gens!), tandis que, sur l'autre plateau, instruits et forts qu'ils sont "d'un dogme révélé », leurs successeurs chrétiens leur donneront " un nom dévalorisant... ». Il ne dit pas lequel, mais c'est celui de «païen », assurément. Voilà que le "paganisme» ne signifierait plus "intrication de deux administrations »! Est-ce que cela ne signifie pas que, dans l'esprit de l'A., il n'y aurait pas de religion (je veux dire que ce concept ne serait pas formé) si la pensée sur la surnature n'était pas caractérisée par une révélation et ne méritait pas le nom de " foi »? Faute de quoi tout ne serait que triste bricolage. Pire, un puits sans fond de métaphysique stupide où les Grecs se seraient engloutis... Si l'on trouve ces mots un peu forts, il n'est pour se convaincre de leur exactitude que de lire la suite. En gros, l'A. fait au polythéisme le reproche de n'être pas un monothéisme à dogme. Lisons: "Le paganisme est... un fatras d'impressions, d'interrogations, d'indignations, mais aussi de soumissions ». Soumission, vous avez bien $\mathrm{lu}^{13}$. Une puissance (anonyme) obligerait-elle les «masses " grecques à penser, à faire, à prier, à sacrifier... (j'évite pourtant le : «à croire»). Je ne comprends pas ce que désigne ce «fatras d'impressions» dans la 
religion grecque. Et les interrogations, les indignations? Il en existe, bien sûr. Et l'on voit clairement d'où les tire l'A. ; il n'est pour cela que de consulter l'index pour relever que la somme des références à Platon et à Socrate écrase toutes les autres par leur nombre et l'intérêt que l'A. leur porte ${ }^{14}$, avec Aristote, Euripide, Hésiode, Homère, Pindare, ils sont vainqueurs toutes catégories. Eu égard à son contenu, ne faudrait-il pas titrer le livre « le paganisme de Platon »? Ou « des philosophes »? Soit à connaître les notions et débats de la religion chrétienne au siècle de Louis XIV, s'il est bien sûr grandement légitime d'y mesurer tout la place prise par le jansénisme et les Jésuites (e.g.), celle des rapports entre l'Église et la monarchie, mais, je dirais, surtout, qu'il est urgent d'enquêter sur la vie chrétienne du paroissien moyen.

Alors que, depuis plus d'un siècle, des historiens spécialisés dans l'étude des religions ont fait tout ce qu'il ont pu pour abandonner le point de vue autocentré et adopter une disposition de l'esprit consistant à s'efforcer de penser dans les mêmes cadres matériels et mentaux que ceux des hommes qui ont vécu ces présents d'autrefois, voilà qu'en 2015 , on nous dit que ce n'est pas le bon moyen, qu'il faut en revenir au regard étranger sur des étrangers. À qui cherche le sens d'une pensée religieuse, quelle autre voix pourtant que celle de l'immersion? Plus, avec J. Rudhardt, on peut aller jusqu'à parler d'empathie envers l'objet d'étude. Si grande est la distance culturelle entre eux et nous qu'elle constitue la principale difficulté, pour appréhender leur façon de penser leurs dieux qui, pour nous, n'existent pas. C'est du dedans et non du dehors qui faut étudier une religion historique. Ce serait à se demander ce que cherchent les ethnologues en s'immergeant au milieu des «païens " pour les connaître au mieux, pourquoi ils ne les étudient pas depuis Paris? Bien sûr, c'est difficile.

Il est grand temps de laisser ce livre à d'autres, qui pourront vérifier s'il peut leur rendre des services, mais les curieux de la singularité de la pensée religieuse grecque ont autre chose à faire.

\section{NOTES}

1. .Preuve, p. 17, par l'A. : il s'est refusé à proposer dans ce livre « un énième dictionnaire de la “Religion grecque" [en réalité, de dictionnaires, il y en a peu en français], mais [il] s'agit de rendre au paganisme ce qu'il fut: l'acceptation... de l'inconnaissance humaine et de l'inconsistance divine». Peu de mots et d'expressions de cette citation vont de soi et correspondent à ce que nous savons de la religion grecque.

2. .Tant pis si, à la page suivante, Mattéi constate que «l'absence de tout credo en matière religieuse rend difficile de parler en termes de "croyance ". » S'est-il interrogé sur les variances du concept de "croyance» d'une religion à une autre? Au passage, dans cette ouverture, le préfacier nous renvoie (s'adressant aux « lecteurs intéressés»), entre autres, au Dictionnaire de l'ésotérisme de Servier et à l'ancêtre, le Dictionnaire des antiquités de Daremberg et Saglio... Merci.

3. .Et si nous ne nous sentons pas membres de ce «nous»? Faut-il un brevet pour continuer la lecture?

4. .Ces italiques sont miens.

5. .Ces guillemets sont miens. 
6. .C'est peut-être en raison de l'usage du mot «paganisme » dans le titre du livre, qui sonne comme une provocation, étant employé pour une époque où le mot et, en Grèce même, la notion, n'existent pas, que l'A. cherche à légitimer cet anachronisme. Mais ce sera moins sur cette utilisation que sur les raisons que l'A. se donne de justifier ce «paganisme » qu'on lui demandera de s'expliquer.

7. .Au sein de ce système d'équivalences, une statue de culte, apparaît, alternativement, sainte et sacrée ici, est une idole, là.

8. .On notera qu'au risque de trahir le sens du mot, on ne saurait écrire «les religions des autres ». Elles sont toutes dans un même sac païen.

9. .Ces guillemets de l'A. disparaîtront chez lui aussitôt. Je les garderai lorsque j'emploierai «paganisme » dans le sens qu'il lui donne.

10. .On sourira à la pensée qu'avec une telle définition le Vatican aussi puisse être qualifié de païen (et bien d'autres constructions politiques du passé et d'aujourd'hui).

11. .On notera que si la question de l'évolution intéresse peu l'A. (pas d'entrée 'histoire'), celle de la géographie non plus. La plupart du temps, le propos est comme suspendu.

12. .Il se réjouit du fait que des commentateurs viendront plus tard - tous moulés dans les catégories du monothéisme - «pour donner un nom dévalorisant à une piété inquiète car précisément démunie de toute certitude» (p. 15). L'image me saisit d'effroi à l'idée de tous ces Grecs inquiets et pourtant pieux ...

13. .L'A. a laissé d'autres traces d'une lecture cynique et «politique » de la religion grecque à propos de l'initiation éleusinienne (sous le sous-titre : Epopteia et démos), il a, par exemple, cette formule: «Avec l'appropriation d'Éleusis, sa récupération, son écho panhellénique, Athènes fonde toutes les convictions [sur la mort...] en un seul espoir : la béatitude de l'initié. Splendide manipulation des masses» (p. 285) («masse», c'est le terme qu'il utilise pour désigner le commun des Grecs). Cette expression, «manipulation », constitue probablement un hapax dans les commentaires! Elle suppose l'existence d'athées cyniques qui ne croient pas à ce qu'ils font et cherchent à en tirer profit, comptant sur l'obscurantisme des fidèles. Dadouques et Kérykes en « manipulateurs » des foules, voilà une représentation originale des rituels éleusiniens.

14. Je laisse de côté le problème posé par la quasi-absence de références épigraphiques ; alors que, par sa variété, sa précision, sa plus grande proximité avec la population, sa diffusion géographique et chronologique, l'épigraphie constitue un si puissant moyen de connaissance des pratiques, mais aussi de la pensée religieuses.

\section{AUTEURS}

\section{PIERRE BRULÉ}

Université de Rennes 2 